\title{
Plumbagin Protects H9c2 Cardiomyocytes against TBHP-induced Cytotoxicity via Alleviating ROS- induced Apoptosis and Inducing Autophagy
}

\section{Qianrui Zhang}

General Hospital of the Yangtze river shippinging

Haitan Fu

General hospital of the Yangtze river shipping

Wenjuan Gong

General hospital of the Yangtze river shipping

\section{Feng Cao}

General hospital of the Yangtze river shipping

Tao Wu ( $\nabla$ hydrinsk@sina.com )

Wuhan fourth hospital https://orcid.org/0000-0003-3638-5283

Fei Hu

General hospital of the Yangtze river shipping

\section{Research Article}

Keywords: Plumbagin, ROS, myocardial injury, apoptosis, autophagy, p38 MAPK

Posted Date: October 20th, 2021

DOl: https://doi.org/10.21203/rs.3.rs-974481/v1

License: (c) (i) This work is licensed under a Creative Commons Attribution 4.0 International License. Read Full License 


\section{Abstract}

Plumbagin has been previously reported to alleviate myocardial ischemia/reperfusion injury in vivo. In this study, we analyzed the potential role of plumbagin against hydrogen peroxide-induced injury in cardiomyocytes. In the present study, plumbagin (PLB) was used to evaluate its cytoprotective property in H9c2 cardiomyocytes against tertiary butyl hydrogen peroxide (TBHP, $75 \mu \mathrm{M})$ induced ROS-mediated oxidative stress and apoptosis. Our results implicate that pretreatment with PLB $(5,10$ or $20 \mu \mathrm{M})$ notably restored viabilities in TBHP-induced $\mathrm{H} 9 \mathrm{c} 2$ cells $(p \otimes 0.01)$. Also PLB treatment significantly decreased creatine kinase (CK) ( $p \bigotimes 0.01)$ and lactate dehydrogenase $(\mathrm{LDH})$ activity $(p \otimes 0.01)$. TBHP induced apoptosis and oxidative stress in cultured cardiomyocyte, whereas PLB pretreatment significantly reduced TBHPinduced apoptosis rate $(p \llbracket 0.01)$ and ROS level $(p \otimes 0.01)$. Furthermore, PLB resulted in decrease in the expressions of cleaved caspase 3 , nicotinamide adenine dinucleotide phosphate (NADPH) oxidase enzyme 4 (NOX4) and phospho-p38 MAPK in TBHP-induced H9c2 cells. And the active marker of autophagosomes, LC3-II/LC3-I was elevated following treatment with PLB. These findings indicated that PLB may induce autophagy. The present study shows the protective role of PLB against TBHP-induced cardiomyocyte injury via alleviation of ROS-mediated apoptosis and induction of autophagy.

\section{Introduction}

According to the Global Burden of Diseases Study 2017, stroke and ischaemic heart disease (IHD) were the leading causes of death in China in 2017 [1]. Acute myocardial infarction is the most serious type of cardiovascular disease. There are more than 3 million patients with acute ST-segment elevation myocardial infarction each year. The most effective treatment for these patients is timely and effective reperfusion therapy [2]. Reperfusion therapy improves myocardial blood supply and is accompanied by a series of pathophysiological reactions, including peroxidation, inflammation, intracellular calcium overload, and finally irreversible apoptosis and necrosis. This myocardial injury caused by reperfusion is called Reperfusion injury [3]. However, the treatment of reperfusion injury is still a clinical problem to be solved urgently.

During myocardial ischemia-reperfusion, myocardial NOX4 expression is up-regulated and activity is enhanced, producing a large amount of ROS, which is an important mechanism leading to myocardial injury [4]. ROS can trigger a variety of signal transduction pathways, including enzyme-coupled receptor signaling pathways and $\mathrm{G}$ protein-coupled receptor signaling pathways, among which mitogen-activated protein kinases (MAPK) signaling pathways play a key role in a large number of cell activities (such as proliferation, differentiation, survival and death) [5]. Inhibition of over-activated p38 MAPK can significantly reduce experimental myocardial ischemia/reperfusion (I/R) injury [6].

Plumbagin (5-hydroxy-2-methyl-naphthalene-1,4-dione) is a major bioactive compound existing in the roots of Plumbago zeylanica. Plumbagin (PLB) not only inhibits platelet aggregation induced by ADP in cardiovascular system, but also suppresses the NOX4 expression, which can significantly improve the redox state imbalance of myocardial ischemia and reperfusion [7-9]. The above studies suggest PLB can 
alleviate myocardial ischemia-reperfusion injury and has great potential for application research in the treatment of cardiovascular diseases. Therefore, this study intends to verify the protective effect of PLB on the oxidative stress and apoptosis of $\mathrm{H} 9 \mathrm{c} 2$ cardiomyocytes induced by tert-butyl hydroperoxide (TBHP).

\section{Materials And Methods}

\section{Materials}

Plumbagin (S4777) was purchased from Selleck Chemicals (Houston, USA). Anti-LC3-II/LC3-I, anti-NOX4, anti-phospho-p38 MAPK and anti-p38 MAPK were from Bioswamp (Wuhan, China). Anti-cleaved caspase 3 was from Abcam (Cambridge, UK). Anti-GAPDH was provided by Abcam (Cambridge, UK).

\section{Cell culture and treatments}

H9c2 cardiomyocytes were obtained from the American Type Culture Collection (ATCC, Manassas, VA, USA). Cells were grown in DMEM (Hyclone, SH30022.01B) requiring supplementation with $10 \% \mathrm{FBS}, 100$ $\mu \mathrm{g} / \mathrm{mL}$ penicillin and $100 \mu \mathrm{g} / \mathrm{mL}$ streptomycin and maintained at $37^{\circ} \mathrm{C}$ in a humidified $5 \% \mathrm{CO}_{2}$ incubator. The confluent cardiomyocytes were cultured in DMEM supplemented with $2 \% F B S$ for another $12 \mathrm{~h}$ before experiment. For experiments, cells were pre-incubated with PLB $(5,10$ or $20 \mu \mathrm{M})$ for $24 \mathrm{~h}$ and TBHP (75 $\mu \mathrm{M})$ for another $4 \mathrm{~h}$. In our study, PLB was dissolved in DMSO and then diluted with DMEM with the final concentration of DMSO less than $0.1 \%$. TBHP was also dissolved in DMEM.

\section{Cell viability assays}

Cardiomyocytes were seeded in 96 -well plates at a density of $5 \times 10^{3}$ cells per well. The cells were pretreated with PLB $(5,10$, and $20 \mu \mathrm{M})$ for $24 \mathrm{~h}$ before being exposure to TBHP for another $4 \mathrm{~h}$. The number of viable cells was determined by a CCK-8 kit. Briefly, the DMEM culture medium was discarded and $100 \mu \mathrm{L}$ CCK-8 reagents (Beyotime Inst, Shanghai, China) were added to a fresh DMEM medium. The 96-well plate was incubated in a carbon dioxide incubator for $2 \mathrm{~h}$. The optical density (OD) values were determined at $450 \mathrm{~nm}$ wave-length. The cell proliferation rate (\%) was calculated as follows: (OD value of experimental well $\triangle \mathrm{OD}$ value of control well)/OD value of control well $\times 100 \%$. The CCK-8 assay was repeated three times for consistency.

\section{LDH and CK leakage}

Cytotoxicity was evaluated by detecting the extent of plasma membrane damage by using commercially available LDH-estimation kit (Beyotime Inst, Shanghai, China) and CK-estimation kit (Beyotime Inst, Shanghai, China). For LDH and CK leakage assays, H9c2 cells were grown in 24-well plates at a density of $3 \times 10^{5}$ cells/well, and the cells were subjected to further experiment after $24 \mathrm{~h}$. The LDH and CK activity were measured after $24 \mathrm{~h}$ of treatment according to the corresponding detection kits.

\section{Estimation of intracellular ROS production}


ROS generation was determined by detecting fluorescence intensity of dichlorofluorescin (DCF) by flow cytometry. Cells were treated with DCFH-diacetate $(10 \mu \mathrm{M})$ at $37^{\circ} \mathrm{C}$ in the dark for $20 \mathrm{~min}$. The cells were then collected and suspended in PBS. The fluorescence intensity of DCF was analyzed by using flow cytometry at an excitation wavelength of $488 \mathrm{~nm}$ and an emission wavelength of $519 \mathrm{~nm}$. Each assay was performed three times.

\section{Measurement of apoptosis}

After the treatment, cardiomyocytes $\left(1.5 \times 10^{5}-1 \times 10^{6}\right)$ were collected and immobilized in $75 \%$ cold ethanol for $12 \mathrm{~h}$ at $4^{\circ} \mathrm{C}$. Then the cells were double-stained with Annexin V-FITC $(10 \mu \mathrm{L})$ and PI $(10 \mu \mathrm{L})$ in the dark at room temperature for $30 \mathrm{~min}$. The apoptotic rate of cardiomyocytes was analyzed by using flow cytometry (BD Biosciences, Franklin lakes, NJ, USA). Each test was repeated three times.

\section{Western blot analysis}

Protein levels were analyzed in whole cardiomyocytes lysates. A total of $30 \mu \mathrm{g}$ samples were separated by $10 \%$ SDS-PAGE and transferred to a PVDF membrane (Millipore, Bedford, MA, USA). The membranes were blocked in $5 \%$ Bovine Serum Albumin for $2 \mathrm{~h}$ and then incubated with the following primary antibodies overnight at $4^{\circ} \mathrm{C}$ (NOX4 1:1000, cleaved caspase-3 1:1000, LC3-II/LC3-I 1:1000, p-p38 MAPK 1:1,000, p38 MAPK 1:1000 and GAPDH 1:1000). The next day, membranes were incubated with secondary antibody at room temperature for $1.5 \mathrm{~h}$. The antibody activity was detected by an ECL method (Beyotime institute of Biotechnology, Shanghai, China).

\section{Statistical analysis}

Data were presented as the mean \pm S.D. Statistical analysis of data was implemented by one-way ANOVA with LSD post hoc analysis. Value of $p$ less than 0.05 were regarded statistically significant.

\section{Results}

\section{Plumbagin protected H9c2 cells from cell death}

Cell viability was measured by using the CCK8 assay as shown in Figure 1A. TBHP significantly reduced cell viability compared with the control group $(p<0.01)$. And PLB $(5,10$ or $20 \mu \mathrm{M})$ pretreatment obviously promoted cell viability compared with the TBHP group $(p<0.01)$.

On the other hand, the elevated LDH and CK activity by TBHP exposure were reduced by PLB pretreatment $(p<0.01$, Figure 1B).

\section{Plumbagin alleviated TBHP induced elevation of ROS production}

The level of ROS in H9c2 cells was quantified by using DCF-DA staining. TBHP treatment significantly increased the intracellular level of ROS in H9c2 cells. And pretreatment with PLB $(5,10$ and $20 \mu \mathrm{M})$ decreased the ROS generation $(p<0.01$, Figure $2(\mathrm{~A}-\mathrm{F}))$. 


\section{Plumbagin prevented TBHP induced apoptosis}

As shown in Figure $3(A-F)$, the apoptotic rate was significantly increased in the TBHP group than in the control group $(p<0.01)$. Pretreatment with PLB $(5,10$ or $20 \mu \mathrm{M})$ significantly reduced the apoptotic rate as compared with TBHP group $(p<0.05)$.

\section{Plumbagin promoted the autophagy of $\mathrm{H} 9 \mathrm{c} 2$ cells}

The level of active marker of autophagosomes, LC3-II/LC3-I were analyzed by using western blot (Fig 4). TBHP notably reduced the ratio of LC3-II/LC3-I, whereas the ratio of LC3-II/LC3-I was obviously promoted by PLB pretreatment as compared with TBHP group. The data indicated that PLB might induce autophagy in TBHP-treated H9c2 cells.

\section{Plumbagin suppressed the NOX4/p38 MAPK pathway}

NOX4, cleaved caspase 3, phospho-p38/p38 MAPK protein expression in TBHP group was significantly increased as compared with control group $(p<0.01)$. Pretreatment with PLB $(5,10$ or $20 \mu \mathrm{M})$ notably suppressed NOX4, cleaved caspase 3 and phospho-p38/p38 MAPK protein expression as compared with the TBHP group ( $p<0.05$ and $p<0.01$, Figure 5).

\section{Discussion}

Myocardial ischemia-reperfusion injury is closely related to oxidative stress. Under physiological conditions, a small amount of oxygen free radicals generated in the body can be quickly eliminated. However, when cells are ischemic and hypoxic, intracellular metabolism is disordered and oxygen free radical scavenging capacity is insufficient. When the ischemic tissue suddenly restores blood supply, a large number of oxygen free radicals are produced, which cannot be cleared in time, causing damage to myocardial tissues and surrounding cells. This causes severe myocardial damage [10-12]. Plumbagin (PLB) is a natural naphthoquinone compound. Existing studies have found that PLB can protect the myocardial damage by modulating the cardiac biomarkers, antioxidants, and apoptotic signaling in the doxorubicin-induced cardiotoxicity in rats [13]. PLB can also inhibit NOX4 and regulate redox signals [14]. Thus, we investigated the effect of PLB on H9c2 cardiomyocyte injury induced by oxidative stress. The experimental doses were based on previous in vitro study [15].

Tertiary butyl hydrogen peroxide (TBHP) is a kind of pro-oxidant, which leads to membrane permeability, lipid peroxidation, ATP consumption, protein thiol group modification and cytoplasmic calcium ion concentration imbalance by generating tert-butoxy groups [16]. TBHP also causes cytotoxicity through the loss of membrane integrity characterized by the release of cytochrome $\mathrm{C}$, increasing p53 expression and mitochondrial membrane transformation, leading to mitochondrial-mediated apoptosis or necrosis [17]. Therefore, this study selected the classic oxidant TBHP to induce oxidative stress damage in the $\mathrm{H} 9 \mathrm{c} 2$ cell line, and explored the cytoprotective effect of plumbagin on TBHP-induced cardiomyocyte injury. Under the mediation of the electron carrier 1-Methoxy PMS, CCK-8 is reduced by dehydrogenase in 
cell mitochondria to form a highly water-soluble yellow formazan. The number of formazan produced is directly proportional to the number of living cells. Therefore, the CCK-8 measurement can indirectly reflect the number of living cells. The results of this study showed that TBHP reduced the proliferation rate of H9c2 cells, suggesting that TBHP-induced oxidative stress caused cell viability to decrease. Pretreatment with plumbagin can reduce the negative impact of TBHP on cell proliferation and improve cell viability in a dose-dependent manner. Lactate dehydrogenase (LDH) and creatine kinase (CK) are important indicators for the diagnosis of acute myocardial infarction, angina pectoris, myocarditis and other myocardial injury. After ischemia and reperfusion, the myocardial cell membrane is damaged and the permeability increases, which causes the leakage of intracellular lactate dehydrogenase (LDH) and other enzymes, and the level of LDH in plasma increases. CK is an important enzyme in the body's energy metabolism. When various tissues and organs of the body are diseased, especially myocardial cell membrane damage caused by myocardial ischemia, the intracellular CK leaks, and the vitality of the cells is reduced [18]. Plumbagin pretreatment can reduce LDH and CK activity induced by TBHP, showing that it can improve TBHP-induced cardiomyocyte damage.

The excessively produced ROS during the ischemia-reperfusion period leads to oxidative stress, which is an important pathogenic factor of myocardial ischemia-reperfusion injury [19]. Under physiological conditions, there is a certain amount of ROS in the myocardium. When myocardial ischemia and hypoxia occur, the function of ROS scavenging system decreases and the function of generating system increases. Once the blood oxygen supply is restored, ROS will be produced in large quantities and accumulate sharply, causing acute or chronic cardiomyocytes injury [20]. During myocardial ischemia and reperfusion, ROS can be produced in large quantities through the myocardial cell mitochondria, vascular endothelial cell purine oxidase and other oxidases, neutrophil respiration burst, catecholamines and other pathways to make the myocardial cell membrane and subcellular organelle membrane fluidity and communication. Increased permeability and loss of integrity result in membrane dysfunction of ion transport, a large amount of $\mathrm{Ca}^{2+}$ floods into cells, and ROS can directly attack cell structural proteins and nucleic acids. The results of this study showed that TBHP significantly increased the intracellular ROS level of H9c2 cells. However, the production of ROS was reduced after PLB pretreatment.

Apoptosis is one of the main pathogenic mechanisms of ischemia-reperfusion injury [21, 22]. Oxidative stress leads to changes in the metabolic and functional properties of mitochondria in ischemic myocardium, thus activates the mitochondrial apoptotic pathway. ROS causes oxidative damage to membrane proteins and lipids, leading to mitochondrial dysfunction, releasing cytochrome $\mathrm{c}$ and ultimately activating caspase, especially caspase-3, all of these can induce apoptosis [23]. In this study, we found that PLB reduced TBHP-induced apoptosis of H9c2 cells by reducing lysed caspase 3 , which indicated that PLB inhibited apoptosis by inhibiting the intrinsic apoptotic pathway mediated by mitochondria.

Autophagy is a ubiquitous protein degradation process, and its role is to remove abnormal proteins and organelles, and realize energy recycling [24]. The specific process is divided into: Induction of macroautophagy, Formation of the autophagosome, Autophagosome docking and fusion, and 
Autophagic body breakdown [25]. Autophagy occurs at a basic level for sustained metabolic recycling of intracellular components in most tissues. Under pathological conditions, autophagy can act as a cytoprotective mechanism to degrade and recycle defective cytoplasmic proteins [26]. Dramatically, inhibition of autophagy causes adverse effects in cardiomyocytes [27]. Also the dual effect of autophagy in cardiovascular disease has been discussed. An increasing number of investigations have confirmed that autophagy has obvious effects on the stimulation of the inflammatory responses and is responsible for the formation of ceroids in atherosclerosis [28]. Microtubule-associated protein 1 light chain 3 (LC3) plays key role in autophagosome formation during autophagy. Activated LC3-I can conjugate with the target lipid phosphatidylethanolamine (PE) on the outer membrane, forming LC3-II. Finally LC3-II is cleaved to LC3-I and released back to the cytosol or degraded upon autophagosome maturation [29]. Thus, the expression of LC3-II/LC3-I has been regarded as a classic autophagy marker. The fact that PLB induced autophagy in $\mathrm{H} 9 \mathrm{c} 2$ cells was confirmed by Western blot analysis, which showed that PLB increased the LC3-II/LC3-I ratio. There is a cross-talk between apoptosis (type 1 cell death) and autophagy (type 2 cell death) in heart injury. When autophagy is promoted, cell death induced by apoptosis will be inhibited [30]. This is in coincidence with our results: TBHP enhanced apoptosis in H9c2 cells, and PLB abolished this when autophagy was induced.

Mitogen-activated protein kinase (MAPK) activation constitutes a pattern of intracellular signaling. Studies have shown that MAPK is involved in myocardial ischemia-reperfusion injury [31]. The p38 mitogen-activated protein kinase (p38 MAPK) pathway regulates the response of cells to growth, apoptosis and stress signals in different cell models. Phosphorylation of threonine and tyrosine residues in p38 leads to conformational changes, thereby increasing the accessibility of the active site and enhancing the catalysis [32]. Some researchers have suggested that autophagy can be induced by activating p38 MAPK, and upregulating autophagy via the p38 MAPK pathway could protect H9c2 cells from oxidative stress $[33,34]$. Besides, the downstream role of the p38 MAPK pathway depends on the assembly of Nox subunits into the NAPDH oxidase complex responsible for ROS production [35]. Considering that ROS can activate 38 MAPK phosphorylation involved in cytotoxicity, this study evaluated the effect of PLB treatment on p38 MAPK phosphorylation. Compared with TBHP-only group, PLB treatment significantly reduced TBHP-induced phosphorylation of p38 MAPK. The results showed that PLB reduced p38 MAPK phosphorylation in H9c2 cells induced by TBHP.

Scavenging ROS failed to effectively prevent the progression of cardiovascular disease [36]. Large-scale multi-center clinical studies (HOPE, SECURE, GISSI and HPS) did not confirm the therapeutic value of vitamin $\mathrm{E}$ (a free radical scavenger) in slowing atherosclerosis and reducing major cardiovascular events. This may be due to the fact that simply giving antioxidant vitamins failed to eliminate the root cause of a large amount of ROS-oxidase, especially NOX [37]. The NOX family is the homolog of NADPH oxidase, which exists on the plasma membrane of different non-phagocytic cells, and the tissue expression has certain specificity. Among them, Nox 1 and Nox 5 are mainly expressed in smooth muscle and endothelial cells, respectively. Nox 2 is found in endothelial cells and adventitia fibroblasts and vascular smooth muscle cells. Similarly, Nox4 is detected in all the above cell types, which is the main source of intracellular ROS [38]. In addition, the expression and activity of NOX4 in myocardial ischemia and 
reperfusion myocardium are up-regulated and the activity is enhanced, producing a large amount of ROS, which is an important mechanism leading to myocardial injury [39]. The results of this study indicated that plumbagin suppressed the expression of NOX4 induced by TBHP in H9c2 cells, which may help reduce the production of ROS.

Overall, we found that plumbagin alleviated TBHP-induced cytotoxicity by reducing ROS-induced apoptosis and inducing autophagy in cardiomyocytes.

\section{Declarations}

\section{Funding}

This work was supported by Science and Technology Project of the Changjiang River Administration of Navigational Affairs (201810010).

\section{Compliance with ethical standards}

\section{Conflicts of interest}

All the authors declared that they have no conflict of interest. No human or animal was used in this study.

\section{References}

1. Zhou M, Wang H, Zeng X et al (2019) Mortality, morbidity, and risk factors in China and its provinces, 1990-2017: a systematic analysis for the Global Burden of Disease Study 2017. Lancet 394(10204):11451158.

2. Arslan F, Bongartz L, Ten Berg JM et al (2018) 2017 ESC guidelines for the management of acute myocardial infarction in patients presenting with ST-segment elevation: comments from the Dutch ACS working group. Neth Heart J 26(9):417-421.

3. Neri M, Riezzo I, Pascale N et al (2017) Ischemia/Reperfusion Injury following Acute Myocardial Infarction: A Critical Issue for Clinicians and Forensic Pathologists. Mediators Inflamm 2017:7018393.

4. Siu KL, Lotz C, Ping P et al (2015) Netrin-1 abrogates ischemia/reperfusion-induced cardiac mitochondrial dysfunction via nitric oxide-dependent attenuation of NOX4 activation and recoupling of NOS. J Mol Cell Cardiol 78:174-185.

5. Kim EK, Choi EJ (2010) Pathological roles of MAPK signaling pathways in human diseases. Biochim Biophys Acta 1802(4):396-405.

6. Zhou QL, Teng F, Zhang YS (2018) FPR1 gene silencing suppresses cardiomyocyte apoptosis and ventricular remodeling in rats with ischemia/reperfusion injury through the inhibition of MAPK signaling pathway. Exp Cell Res 370(2):506-518. 
7. Zhang Q, Liao X et al (2017) The naphthoquinone plumbagin suppresses ADP-induced rat platelet aggregation through P2Y1-PLC signaling pathway. Pak J Pharm Sci 30(2(Suppl.)):573-578.

8. Guida M, Maraldi T, Resca E et al (2013) Inhibition of nuclear Nox4 activity by plumbagin: effect on proliferative capacity in human amniotic stem cells. Oxid Med Cell Longev 2013:680816.

9. Wang SX, Wang J, Shao JB (2016) Plumbagin Mediates Cardioprotection Against Myocardial Ischemia/Reperfusion Injury Through Nrf-2 Signaling. Med Sci Monit 22:1250-1257.

10. Zorov DB, Juhaszova M, Yaniv Y et al (2009) Regulation and pharmacology of the mitochondrial permeability transition pore. Cardiovasc Res 83(2):213-225.

11. Kurian GA, Rajagopal R, Vedantham S et al (2016) The Role of Oxidative Stress in Myocardial Ischemia and Reperfusion Injury and Remodeling: Revisited. Oxid Med Cell Longev 2016:1656450.

12. Granger DN, Kvietys PR (2015) Reperfusion injury and reactive oxygen species: The evolution of a concept. Redox Biol 6:524-551.

13. Li Z, Chinnathambi A, Ali Alharbi S et al (2020) Plumbagin protects the myocardial damage by modulating the cardiac biomarkers, antioxidants, and apoptosis signaling in the doxorubicin-induced cardiotoxicity in rats. Environ Toxicol 35(12):1374-1385.

14. Ding Y, Chen ZJ, Liu S et al (2005) Inhibition of Nox-4 activity by plumbagin, a plant-derived bioactive naphthoquinone. J Pharm Pharmacol 57(1):111-116.

15. Zhang Q, Zhao S, Zheng W et al (2020) Plumbagin attenuated oxygen-glucose deprivation/reoxygenation-induced injury in human SH-SY5Y cells by inhibiting NOX4-derived ROSactivated NLRP3 inflammasome. Biosci Biotechnol Biochem 84(1):134-142.

16. Chang G, Zhang D, Yu H et al (2013) Cardioprotective effects of exenatide against oxidative stressinduced injury. Int J Mol Med 32(5):1011-1020.

17. $T$ MM, Anand T, Khanum F (2018) Attenuation of cytotoxicity induced by tBHP in H9C2 cells by Bacopa monniera and Bacoside A. Pathophysiology 25(2):143-149.

18. Callegari GA, Novaes JS, Neto GR et al (2017) Creatine Kinase and Lactate Dehydrogenase Responses after Different Resistance and Aerobic Exercise Protocols. J Hum Kinet 58:65-72.

19. Inafuku H, Kuniyoshi Y, Yamashiro S et al (2013) Determination of oxidative stress and cardiac dysfunction after ischemia/reperfusion injury in isolated rat hearts. Ann Thorac Cardiovasc Surg 19(3):186-194.

20. Ruiz-Ginés JA, López-Ongil S, González-Rubio M et al (2000) Reactive oxygen species induce proliferation of bovine aortic endothelial cells. J Cardiovasc Pharmacol 35(1):109-113. 
21. Jeremias I, Kupatt C, Martin-Villalba A et al (2000) Involvement of CD95/Apo1/Fas in cell death after myocardial ischemia. Circulation 102(8):915-920.

22. McClintock DS, Santore MT, Lee VY et al (2002) Bcl-2 family members and functional electron transport chain regulate oxygen deprivation-induced cell death. Mol Cell Biol 22(1):94-104.

23. Bi YM, Wu YT, Chen $L$ et al (2018) 3,5-Dicaffeoylquinic acid protects H9C2 cells against oxidative stress-induced apoptosis via activation of the PI3K/Akt signaling pathway. Food Nutr Res 62:10.29219/fnr.v62.1423.

24. Maiuri MC, Zalckvar E, Kimchi A et al (2007) Self-eating and self-killing: crosstalk between autophagy and apoptosis. Nat Rev Mol Cell Biol 8(9):741-752.

25. Li L, Tan J, Miao Y et al (2015) ROS and Autophagy: Interactions and Molecular Regulatory Mechanisms. Cell Mol Neurobiol 35(5):615-621.

26. Kim I, Rodriguez-Enriquez S, Lemasters JJ (2007) Selective degradation of mitochondria by mitophagy. Arch Biochem Biophys 462(2):245-253.

27. Gottlieb RA, Mentzer RM (2010) Autophagy during cardiac stress: joys and frustrations of autophagy. Annu Rev Physiol 72:45-59.

28. Hassanpour M, Rahbarghazi R, Nouri M et al (2019) Role of autophagy in atherosclerosis: foe or friend? J Inflamm (Lond) 16:8.

29. He C, Klionsky DJ (2009) Regulation mechanisms and signaling pathways of autophagy. Annu Rev Genet 43:67-93.

30. Nishida K, Yamaguchi O, Otsu K (2008) Crosstalk between autophagy and apoptosis in heart disease. Circ Res 103(4):343-351.

31. Tao H, Nuo M, Min S (2018) Sufentanil protects the rat myocardium against ischemia-reperfusion injury via activation of the ERK1/2 pathway. Cytotechnology 70(1):169-176.

32. Treusch S, Albert FW, Bloom JS et al (2015) Genetic mapping of MAPK-mediated complex traits Across S. cerevisiae. PLoS Genet 11(1):e1004913.

33. Zheng YH, Tian C, Meng Y et al (2012) Osteopontin stimulates autophagy via integrin/CD44 and p38 MAPK signaling pathways in vascular smooth muscle cells. J Cell Physiol 227(1):127-135.

34. Lv XC, Zhou HY (2012) Resveratrol protects H9c2 embryonic rat heart derived cells from oxidative stress by inducing autophagy: role of p38 mitogen-activated protein kinase. Can J Physiol Pharmacol 90(5):655-662. 
35. Tormos AM, Taléns-Visconti R, Nebreda AR et al (2013) p38 MAPK: a dual role in hepatocyte proliferation through reactive oxygen species. Free Radic Res 47(11):905-916.

36. Harrison DG, Gongora MC, Guzik TJ et al (2007) Oxidative stress and hypertension. J Am Soc Hypertens 1(1):30-44.

37. Schramm A, Matusik P, Osmenda G et al (2012) Targeting NADPH oxidases in vascular pharmacology. Vascul Pharmacol 56(5-6):216-231.

38. Takac I, Schröder K, Brandes RP (2012) The Nox family of NADPH oxidases: friend or foe of the vascular system? Curr Hypertens Rep 14(1):70-78.

39. Cadenas S (2018) ROS and redox signaling in myocardial ischemia-reperfusion injury and cardioprotection. Free Radic Biol Med 117:76-89.

\section{Figures}

A

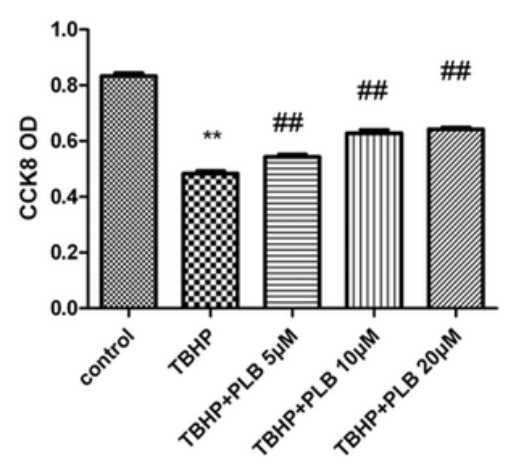

B

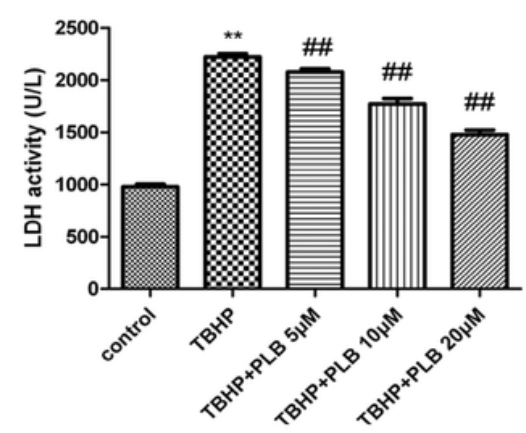

C

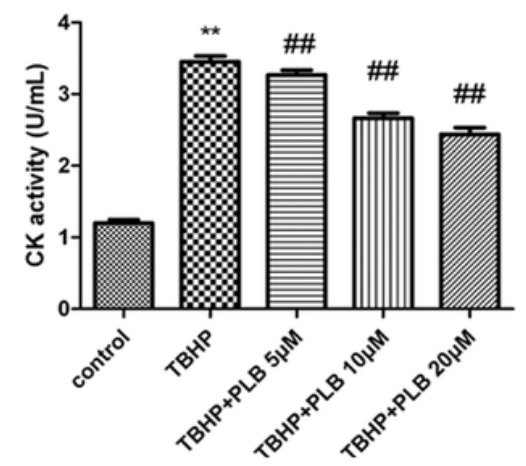

\section{Figure 1}

Plumbagin protected H9c2 cells from cell death. (A) Cell viability; (B) LDH activity; (C) CK activity. Data are expressed as mean \pm S.D. $(n=6)$. ${ }^{\star \star} p<0.01$ vs. the control; $\# \# p<0.01$ vs. the TBHP. (One-way ANOVA with LSD post hoc analysis). 
A

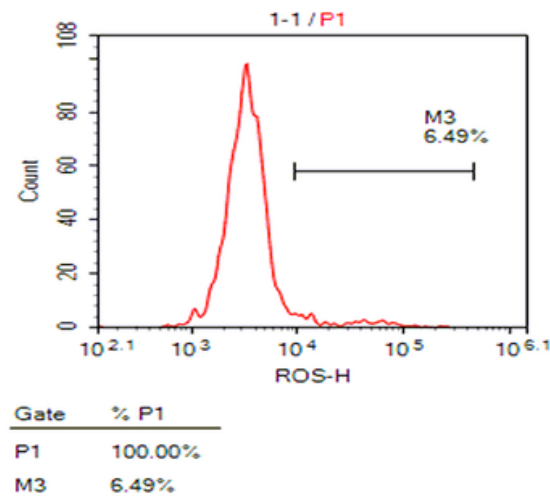

D

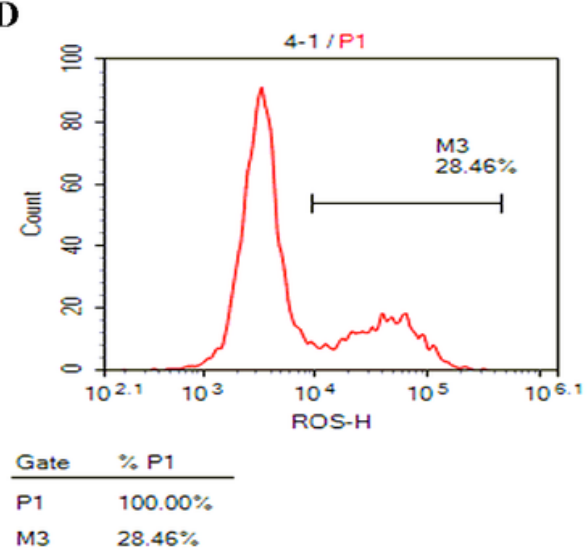

B

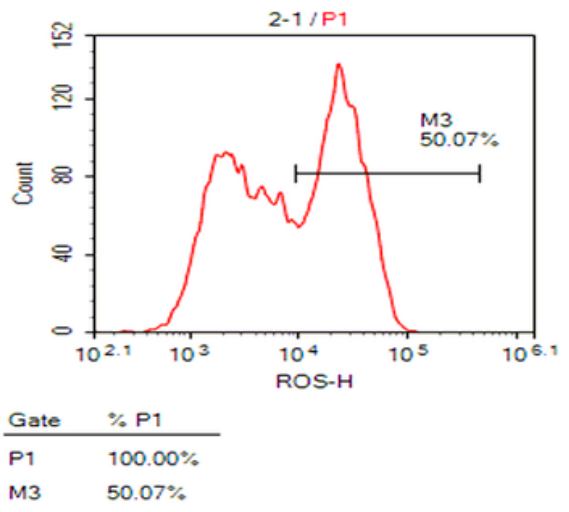

$\mathbf{E}$

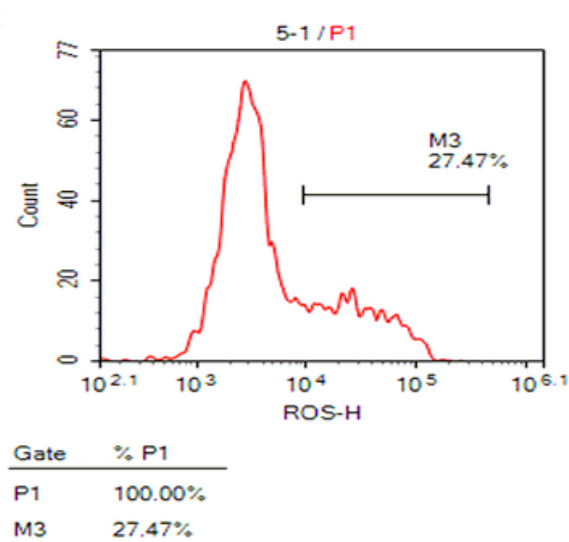

C

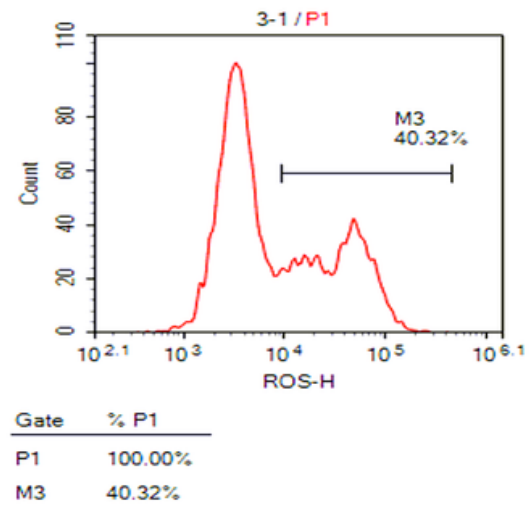

F

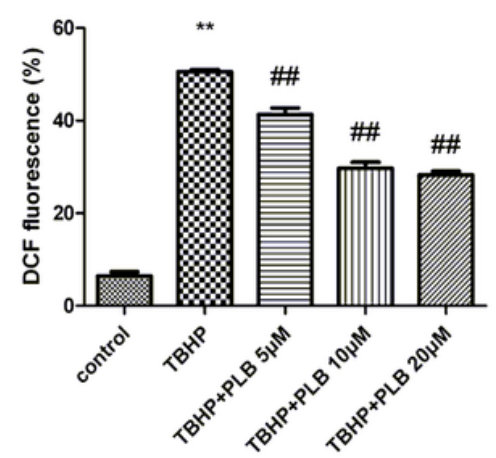

Figure 2

Plumbagin reduced ROS production. ROS level were determined by using DCFH-DA. (A) Control. (B) TBHP. (C) TBHP+PLB $(5 \mu \mathrm{M})$. (D) TBHP+PLB $(10 \mu \mathrm{M})$. (E) TBHP+PLB $(20 \mu \mathrm{M})$. (F) Bar graph represents DCF fluorescence intensity. Data are expressed as mean \pm S.D. $(n=3)$. ${ }^{\star} p<0.01$ vs. the control; \#\#p $<0.01$ vs. the TBHP. (One-way ANOVA with LSD post hoc analysis). 
A

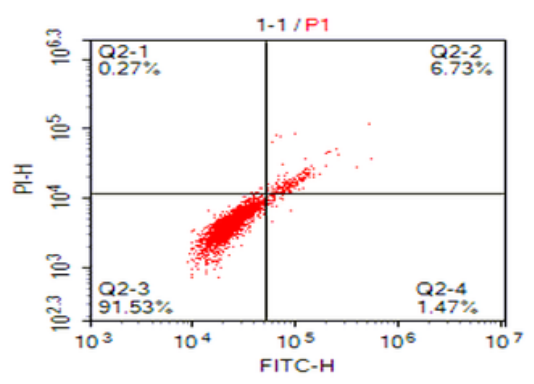

D

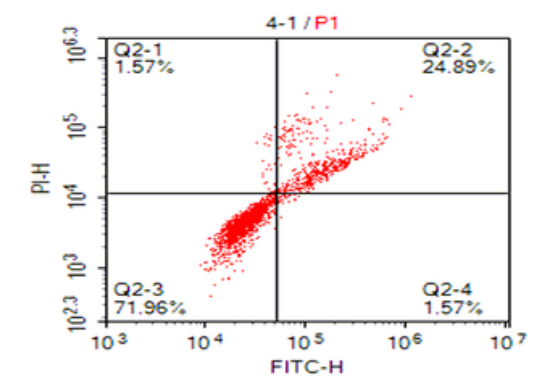

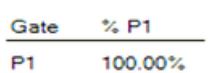

$\begin{array}{ll}\text { P1 } & 100.00 \% \\ \text { Q2-1 } & 1.57 \%\end{array}$

Q2-2 24.89\%

Q2-3 $71.96 \%$

Q2-4 $1.57 \%$

B

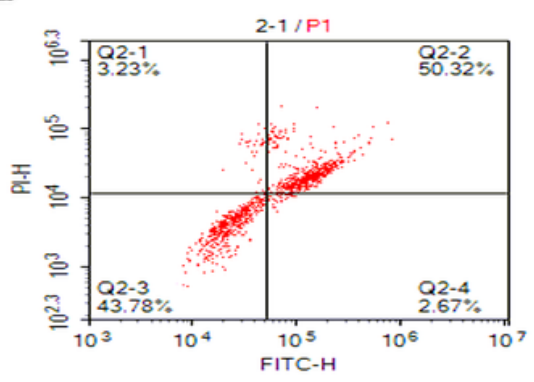

\begin{tabular}{ll} 
Gate & $\% \mathrm{P} 1$ \\
\hline $\mathrm{P} 1$ & $100.00 \%$
\end{tabular}

Q2-1 $3.23 \%$

Q2-2 50.32\%

Q2-3 $43.78 \%$

Q2-4 2.67\%

E

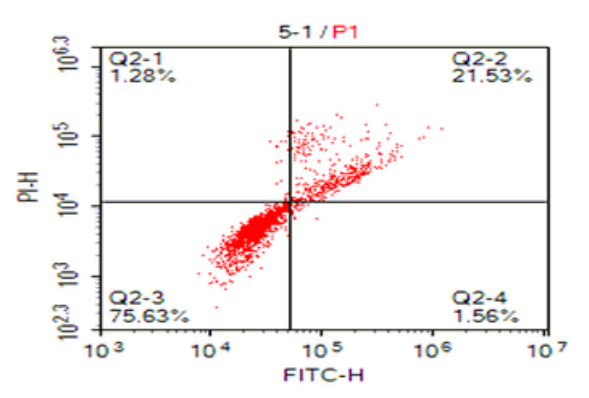

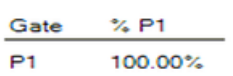

Q2-1 $1.28 \%$

Q2-2 21.53\%

Q2-3 $75.63 \%$

Q2-4 $1.56 \%$
C

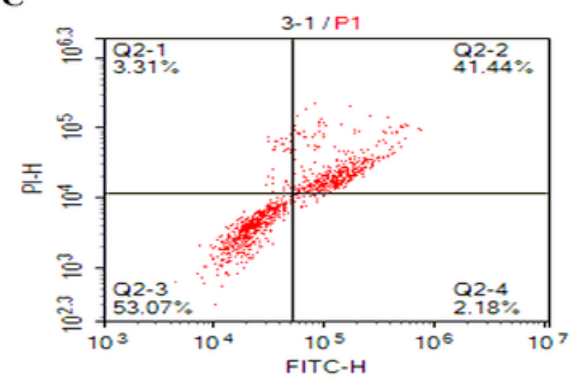

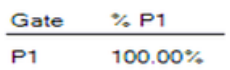

Q2-1 3.31\%

Q2-2 $41.44 \%$

Q2-3 53.07\%

Q2-4 2.18\%

F

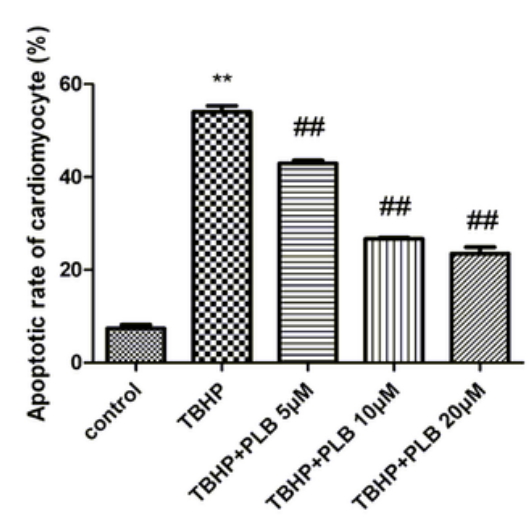

Figure 3

Plumbagin prevented TBHP induced apoptosis. Apoptotic rates were detected by Annexin V-FITC/PI

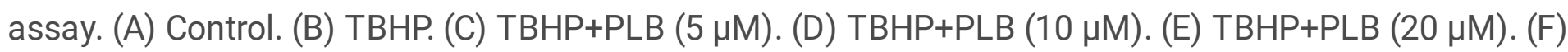
Bar graph represents apoptotic rates. Data are expressed as mean \pm S.D. $(n=3) .{ }^{*} p<0.01$ vs. the control; $\# \#$ p 0.01 vs. the TBHP. (One-way ANOVA with LSD post hoc analysis). 


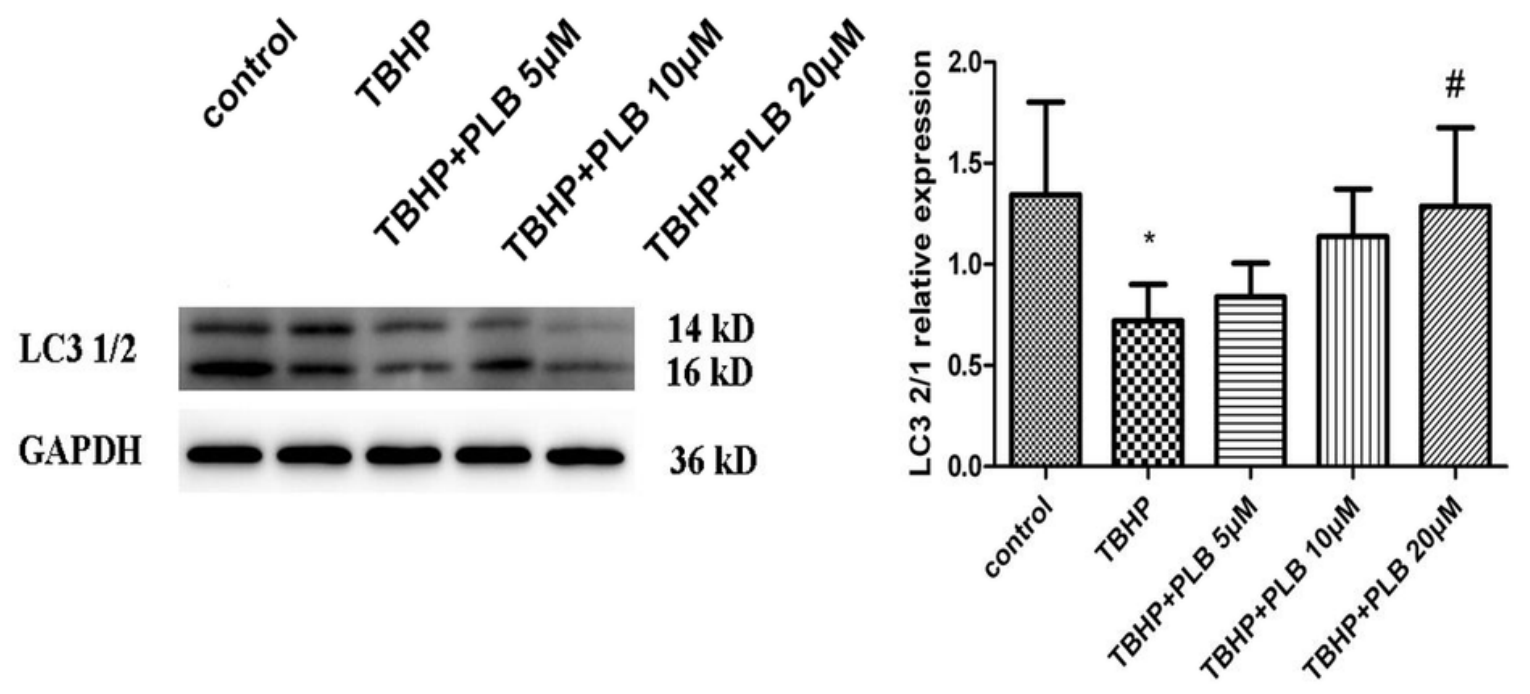

Figure 4

Plumbagin promoted the autophagy of H9c2 cells. (A) Representative western blots for LC3-II/LC3-I; (B) Densitometric analysis for the ratio of LC3-II/LC3-I. Data are expressed as mean \pm S.D. $(n=3) .{ }^{*} p<0.05$ vs. the control; \#p < 0.05 vs. the TBHP. (One-way ANOVA with LSD post hoc analysis).

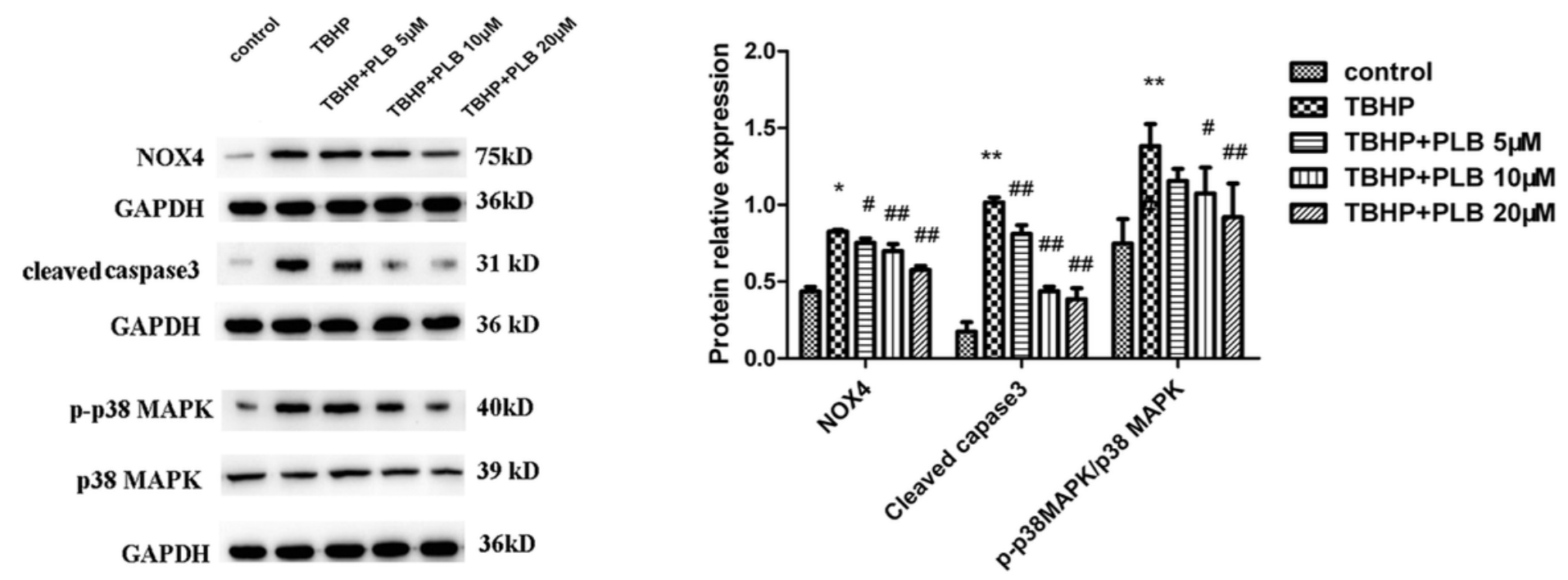




\section{Figure 5}

Plumbagin suppressed the NOX4/p38 MAPK pathway. (A) Representative western blots for NOX4, cleaved caspase 3, phospho-p38/p38 MAPK protein; (B) Densitometric analysis for the expression of NOX4, cleaved caspase 3, phospho-p38/p38 MAPK protein. Data are expressed as mean \pm S.D. $(n=3)$. * $p<$ 0.05 and $* * p<0.01$ vs. the control; $\# p<0.05$ and \#\#p $<0.01$ vs. the TBHP. (One-way ANOVA with LSD post hoc analysis). 